\title{
Travel-time perturbations due to internal waves: Equivalence of modal and ray solutions
}

\author{
Peter Traykovski \\ Massachusetts Institute of Technology/Woods Hole Oceanographic Institution Joint Program in \\ Oceanography and Oceanographic Engineering, Woods Hole, Massachusetts 02453
}

(Received 31 October 1994; accepted for publication 7 September 1995)

\begin{abstract}
In a recent paper, Lynch et al. used modal and ray based perturbation techniques to compare predicted variances of acoustic travel times due to internal waves to measured variances in the Barents Sea Polar Front experiment [Lynch et al., J. Acoust. Soc. Am. 99, 803-821 (1996)]. One of the interesting results of this work is that the modal and ray travel-time variances are substantially different for rays and modes with the same grazing angle. Specifically, the maximum modal travel-time variance shows a resonant effect in which the variance increases with increasing frequency. Unlike the modal solution, the ray travel-time variance has a geometrically constrained maximum, independent of frequency. In this paper, the linear first-order solutions for the ray and modal variances due to the internal waves are reviewed, and in an Appendix the effects of the linearizing assumptions are examined. The ray and mode solutions are then shown to be consistent by considering a truncated sum of modes that constructively interfere along a geometric ray path. By defining the travel-time perturbation due to a truncated sum of modes, the travel-time variance of the modal sum is derived. With increasing frequency the maximum value of this variance converges to a frequency-independent result with a similar magnitude to the ray maximum variance. (C) 1996 Acoustical Society of America.
\end{abstract}

PACS numbers: 43.30.Bp, 43.30.Cq, 43.30.Re

\section{INTRODUCTION}

Internal waves and internal tides are important components of shallow-water coastal oceanography. Whenever a sufficient vertical density gradient exists, oscillations restored by buoyancy can occur. Internal waves and tides are characterized by temperature and velocity fluctuations with periods of tens of seconds to several hours, and are important mechanisms for mixing and energy transport in both the shallow and deep ocean. ${ }^{1}$ Acoustically, the temperature fluctuations cause changes in sound speed, which in turn lead to fluctuations in pulse travel times. In Sec. I of this paper the method for determining the vertical displacement of water caused by the internal waves will be discussed.

Many techniques are available to study acoustic variability associated with internal waves. In the deep ocean, the ideas of ray theory have proven to be a useful tool. ${ }^{2}$ In shallow water, Essen $^{3}$ performed an analysis of phase variability based on modal perturbations. In a recent paper Lynch et al. ${ }^{4}$ have used both modal and ray based perturbation techniques to compare predicted travel-time variance results to measured variances in the Barents Sea Polar Front (BSPF) experiment. One of the interesting aspects of this paper is that the modal and ray travel-time variances are substantially different for rays and modes with the same grazing angle. In Sec. II of this paper, the results of Lynch et al. ${ }^{4}$ are reviewed and the differences between the ray and modal pictures are examined in detail.

Tindle and Guthrie ${ }^{5}$ have shown that a group of modes can constructively interfere to form a ray path. This interference pattern is examined in Sec. III for the rays that are most perturbed by the internal waves. In Secs. IV and V the traveltime variance of the sum of modes that converge along a ray path is shown to be equivalent to the ray travel-time variance.

\section{INTERNAL WAVES}

The vertical displacement due to internal waves at a single frequency can be expressed in terms of a vertical modal structure and horizontally propagating free-wave solutions:

$$
\xi(x, y, z, t)=\sum_{n} \Psi_{n}(z) \exp \left\{i\left(k_{n} x+l_{n} y-\omega t\right)\right\} .
$$

From the linearized equations of motion an eigenvalue equation for the vertical mode functions is derived in many basic oceanography texts ${ }^{6}$ as

$$
\begin{aligned}
& \frac{\partial^{2} \psi_{n}}{\partial z^{2}}+k_{h n}^{2}\left[\frac{N^{2}-\omega^{2}}{\omega^{2}-f^{2}}\right] \psi_{n}=0, \\
& k_{h n}^{2}=k_{n}^{2}+l_{n}^{2}
\end{aligned}
$$

with boundary conditions of $\psi(0)=\psi(D)=0$, and mode normalization given by $\int_{0}^{D} \psi_{n}^{2}(z) d z=1$. Here $f$ is the Coriolis frequency, and the buoyancy frequency profile is described by

$$
N^{2}(z)=-\frac{g}{\rho_{0}} \frac{d}{d z} \rho(z),
$$

where the $z$ axis is positive upward.

The eigenvalue Eq. (2) is discretized and solutions for the eigenvalues and mode shapes are found by using a numerical finite difference approach. 
The oceanographic environment is modeled by a canonical three layer shallow-water system with a warm mixed layer of 20-m depth overlying a cooler layer with a maximum depth of $D=120 \mathrm{~m}$. Between the two regions there is a 20-m-thick transitional layer in which the sound speed and the density vary linearly from the upper to the lower region (Figs. 1 and 2). This is similar to many of the measured profiles in the BSPF experiment. The bottom is assumed to be a perfect acoustic reflector for convenience.

Since the buoyancy frequency $(N)$ is proportional to the $z$ derivative of density, $N$ has step changes (Fig. 1). In the discretization of the eigenvalue equations the step interfaces are handled by enforcing continuity of pressure and vertical velocity across the interface. Although propagating solutions of Eq. (2) can exist for all frequencies between $f$ and $N$, for simplicity only one frequency (the M2 internal tide) is considered here. This tidal frequency has a period of $12.42 \mathrm{~h}$, which is slightly less than the Coriolis period at this latitude. It was found to contain considerable energy in the BSPF experimental region. At this time it is not known what percentage of the energy in the tidal peak in the internal wave spectrum at the BSPF site is due to actual internal tides or to nonlinear higher frequency solitons that are generated each tidal period. This has important effects on the acoustic propagation modelling. The internal tides have long horizontal wavelengths compared to the acoustic modal cycle distance. Thus coupling between acoustic modes will be minimal, and adiabatic propagation can be assumed. However, if the internal waves are solitons with much shorter horizontal wavelengths, significant acoustic mode coupling may occur. For the purposes of this paper it is assumed the peak in the internal wave spectrum is due to internal tides and not solitons, and only adiabatic acoustic modal propagation is considered (see Lynch et al. for more details).

Once the internal wave mode shapes have been calculated the modal amplitudes must be found in order to determine the vertical displacement field. In general the modal amplitudes are functions of both frequency and direction of internal wave propagation. For simplicity only one frequency and one direction are considered here. The direction of the internal wave propagation is set perpendicular to the direc-

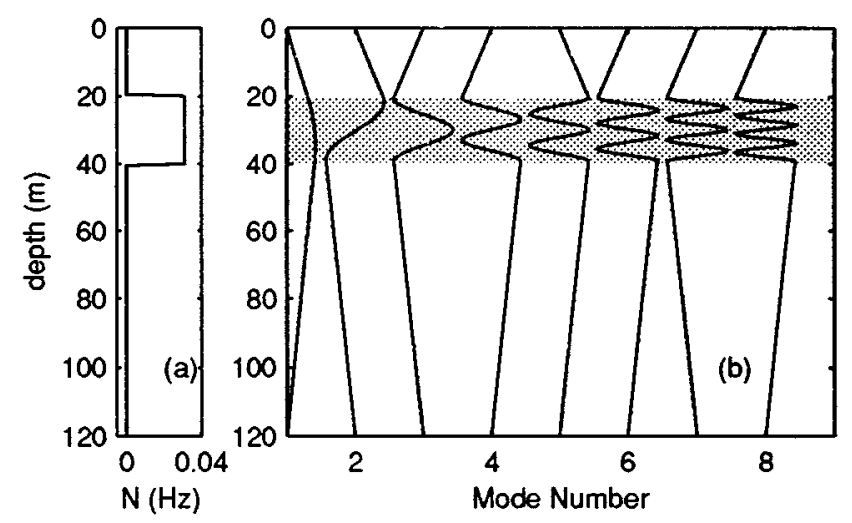

FIG. 1. (a) Buoyancy profile. (b) Internal wave mode shapes. The grey shaded region is the depth interval of nonzero sound-speed gradient and nonzero buoyancy frequency. This is the only region that supports propagating internal waves. tion of acoustic propagation. This simplified case accentuates the effects of the internal waves, since this direction results in the largest variability of acoustic travel time (as discussed in Lynch et al.). Unlike the actual environment in the BSPF experiment, the model used here is range independent with the range used in the calculations arbitrarily set to $1 \mathrm{~km}$.

In the BSPF paper by Lynch et al. the internal wave modal amplitudes were found by examining temperature and current fluctuations in an inverse problem formulation. The spectral density of the modal coefficients was modeled as

$$
\begin{aligned}
& \left|A_{n}(\omega)\right|^{2}=C \frac{\omega^{-3 / 2}}{n^{2}+9}, \quad \frac{\omega}{2 \pi}>1 \mathrm{cpd}, \\
& C=7.6 \times 10^{5} \mathrm{~m}^{2} \mathrm{~s}^{-3 / 2}
\end{aligned}
$$

Since most of the energy is contained in the lower modes, only the first eight need to be used in the analysis. This accounts for $80 \%$ of the energy in Eq. (4). The displacements due to the internal waves can now be written as

$$
\xi(z, t)=\sum_{n} A_{n} \psi_{n}(z) \exp \left\{i\left(k_{n} x+l_{n} y-\omega t\right)\right\}
$$

\section{ACOUSTIC TRAVEL-TIME FLUCTUATIONS}

\section{A. Modal perturbations}

In first-order perturbation theory, the travel-time fluctuation of an acoustic normal mode is found by first calculating the small perturbation to the sound-speed profile due to the internal waves and determining the first-order perturbation to the acoustic horizontal wave number. The travel-time perturbation can then be found as a stationary point in the phase integral. ${ }^{4}$ This stationary point is the time domain analog to finding the group velocity of a normal mode. The group velocity of a mode is a frequency-dependent quantity as seen in Eq. (7). Thus these methods are only applicable to narrowband pulses centered at some frequency $\omega_{0}$. Several linearizations are implicit in the application of this method; the impact of these approximations is discussed at length in Appendix B.

Since the internal wave mode amplitudes are assumed to be independent random variables with zero mean, the acoustic travel-time fluctuations also have zero mean. In the BSPF paper a linear expression relating the variance of the $m$ th modal travel-time perturbation to the variance of the $N$ internal wave amplitudes is found as

$$
\left\langle\delta t_{m}^{2}\right\rangle=\sum_{n=1}^{N}\left\langle a_{n}^{2}\right\rangle\left(R \int_{0}^{D} \psi_{n} S_{z} G_{m} d z\right)^{2},
$$

where $R$ is arbitrarily set here to $1 \mathrm{~km}$ and $S_{z}=d c_{p} / d z$ relates the potential sound-speed perturbation to the displacements from the internal waves. This method of relating sound-speed perturbations to vertical displacements is approximate and is further discussed in Appendix B. The internal wave modal amplitude variance $\left\langle a_{n}^{2}\right\rangle$ is assumed to be equal to its spectral density $\left|A_{n}(\omega)\right|^{2}$ and the mean of $a_{n}$ is assumed to be zero.

The acoustic modal function $\left(G_{m}\right)$ as determined from the stationary point and the perturbation method is 

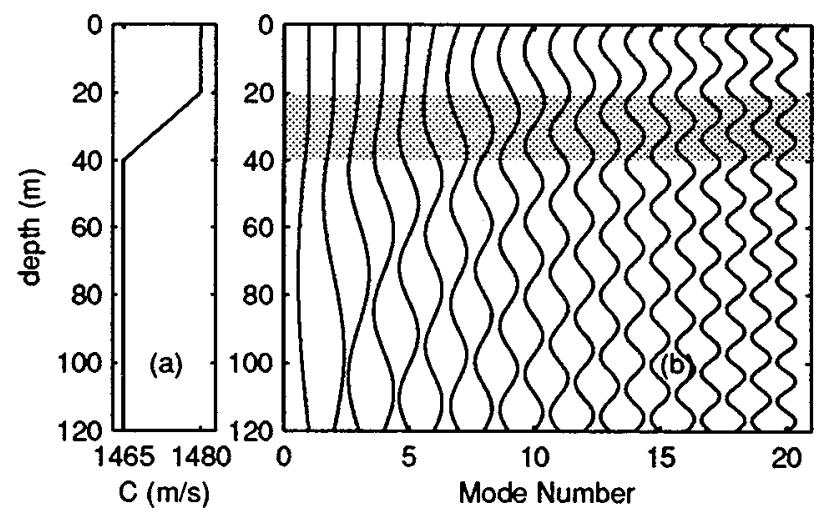

FIG. 2. (a) Sound-speed profile. (b) Acoustic mode shapes for $f=300 \mathrm{~Hz}$. The seventh mode best fits half an oscillation into the gradient layer. This mode experiences the greatest perturbation due to the internal wave displacements.

$$
\begin{aligned}
& G_{m}(z)=\frac{1}{\rho k_{r m} c^{3}}\left[\omega_{0}\left(2-\frac{C_{m}}{U_{m}}\right)\left|\phi_{m}\right|^{2}+\omega_{0}^{2} \frac{\partial}{\partial \omega}\left|\phi_{m}\right|^{2}\right], \\
& C_{m}=\left.\frac{\omega}{k_{r m}}\right|_{\omega=\omega_{0}}, \quad U_{m}=\left.\frac{\partial}{\partial k_{r m}} \omega\right|_{\omega=\omega_{0}},
\end{aligned}
$$

where $k_{r m}$ is the horizontal wave number for the $m$ th acoustic mode. The acoustic mode shapes $\left(\phi_{m}\right)$ are found by a simple numerical finite difference method (Fig. 2). The acoustic modes are normalized by $\int_{0}^{D} \rho^{-1} \phi_{m}^{2} d z=1$. Initially a frequency of $f=300 \mathrm{~Hz}$ is used, but this frequency will be increased in Sec. $\mathrm{V}$ to show equivalence with the ray solution. The sound-speed profile is consistent with the oceanographic model described in Sec. I.

Once the acoustic mode shapes have been determined the derivative of the mode shape function with respect to frequency is calculated by computing the mode shape at a slightly different frequency and then using a finite difference approximation to the derivative. The group velocity of the $m$ th mode $\left(U_{m}\right)$ is calculated using a perturbation approach. ${ }^{7}$ The modal travel-time variance can then be determined from Eq. (6). In Fig. 3 the standard deviations of the travel times are plotted as a function of the angle $\left(\theta_{m}\right)$ associated with each mode. The mode angle is determined from the ratio of the horizontal wave number to the total wave number at the sound-speed minimum. $\theta_{m}=\operatorname{acos}\left(k_{r m} / k\right)$. The mode number is also evident in Fig. 3 since the mode angle monotonically increases with mode number, and the travel-time standard deviations are plotted as discrete points.

For the oceanographic model and internal wave field that have been constructed the maximum travel-time standard deviation of $9.0 \mathrm{~ms}$ occurs for mode number seven with an mode angle of $9.4^{\circ}$. In Fig. 2 it can be seen that modes lower than the seventh have little energy in the region where the sound-speed gradient occurs $\left(S_{z} \neq 0\right)$, and modes greater than the seventh have more oscillations in the region with a nonzero gradient. The higher modes average out the variability associated with the internal waves and are less affected. Thus there is a resonance effect when half of a vertical oscillation best fits the gradient layer. The internal waves cause displacements over the whole water column (except at boundaries) which are greatest in the trapped region of

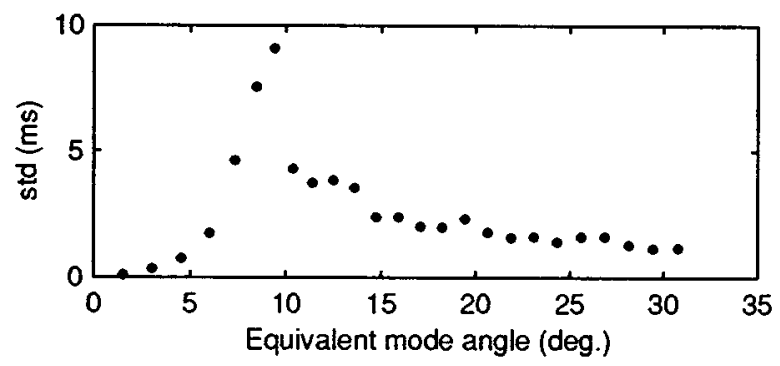

FIG. 3. Standard deviation of modal travel-time perturbations from Eq. (6) evaluated at $f=300 \mathrm{~Hz}, r=1 \mathrm{~km}$, and internal tides at the M2 frequency.

$\omega_{\text {internal wave }}<N$. These displacements can only affect acoustic travel times in regions of nonzero sound-speed gradient, since in other regions the displaced water is isovelocity.

\section{B. Ray perturbations}

The perturbation of travel time along a ray is found in the Lynch et al. BSPF paper by slightly perturbing the sound-speed profile and integrating along the unperturbed path $\left(\Gamma_{0}\right)$. The effects of the unperturbed ray approximation are discussed in Appendix B. The travel-time variance is given by

$$
\left\langle\delta t^{2}\right\rangle=\sum_{n}\left\langle a_{n}^{2}\right\rangle\left(\int_{\Gamma_{0}} \frac{\psi_{n} S_{z}}{c_{0}^{2}} d s\right)^{2} .
$$

The ray paths (Fig. 4) are found by directly integrating the ray equations with a simple numerical integrator. To find the ray with the maximum travel-time variance, rays were calculated with $0.01^{\circ}$ increments between launch angles. In order to find an average travel-time variance at a given range $r$, the travel-time perturbation is determined for one complete ray cycle (range $=R_{c}$ ) and then multiplied by the number of fractions of a ray cycle for the range of interest. $(r=1 \mathrm{~km}$ in this case):

$$
\left\langle\delta t^{2}(r)\right\rangle=\frac{r^{2}}{R_{c}^{2}}\left\langle\delta t^{2}\left(R_{c}\right)\right\rangle .
$$

As can be seen in Fig. 4 at $1 \mathrm{~km}$ the rays have only interacted with the transitional layer once; thus this average is not accurate at such short ranges. For longer ranges with multiple ray cycles this estimate becomes more accurate. This ray average and the modal expression [Eq. (6)] both scale the travel-time standard deviations linearly with respect to range, so it is valid to compare the two quantities.

The maximum travel-time standard deviation of $2.8 \mathrm{~ms}$ occurs at a ray launch angle of $8.1^{\circ}$ (Fig. 5). From the ray trace (Fig. 4) it can be seen that rays with steeper angles than this pass through the transitional layer while flatter rays are refracted off the bottom of the layer. The ray that spends the greatest portion of its cycle distance in the transitional layer experiences the largest perturbation due to the internal waves. The fraction of a cycle distance that this ray spends in the gradient layer is determined by the sound-speed gradient (ray curvature) and the layer thickness. Thus there is a frequency-independent geometric limit to the ray maximum travel-time variance unlike the modal variance which is frequency dependent. 


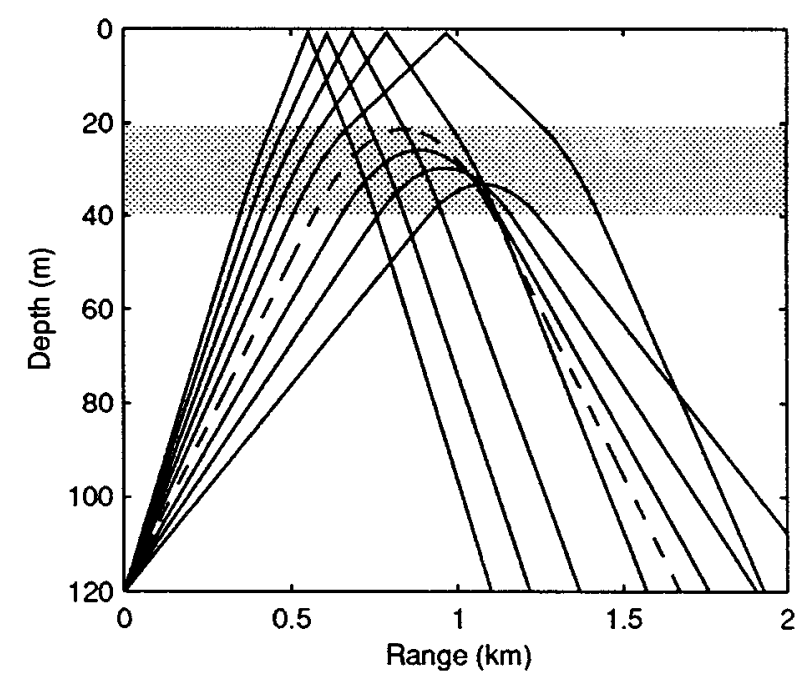

FIG. 4. Ray paths from $5^{\circ}$ to $13^{\circ}$ at $1^{\circ}$ intervals. The dashed line represents the ray with the greatest travel-time variability due to the internal waves.

Comparing the ray results to the modal results at $300 \mathrm{~Hz}$ the maximum modal standard deviation $(9.0 \mathrm{~ms})$ is almost three times the maximum ray standard deviation $(2.8 \mathrm{~ms})$. The modal resonance will be shown to become even larger at higher frequencies in Sec. V. In the following sections this apparent inconsistency is examined in detail by viewing a ray as a finite sum of modes. The mode with the maximum travel-time variance has a grazing angle of $9.4^{\circ}$ while the ray maximum occurs at $8.1^{\circ}$. In the BSPF paper Lynch et al. showed that the grazing angle of the most perturbed mode converges to the ray angle associated with the most perturbed ray at higher frequencies.

\section{MODAL REPRESENTATION OF A RAY}

Tindle and Guthrie have shown that the constructive interference of a group of adjacent normal modes occurs along a ray path. ${ }^{5}$ Here this convergence is examined for the ray that has the largest travel-time variance due to the internal waves. Once the modes that need to be summed to give the best representation of a ray are found, then the travel-time variance for this sum of modes can be examined to see if it approaches the ray travel-time variance.

The truncated modal sum can be written as

$$
p_{\text {ray }}(r, z)=\sum_{m=c-l}^{c+l}\left(\frac{2 \pi}{k_{r m} r}\right) \phi_{m}\left(z_{s}\right) \phi_{m}(z) \exp \left(i k_{r m} r\right) \text {, }
$$

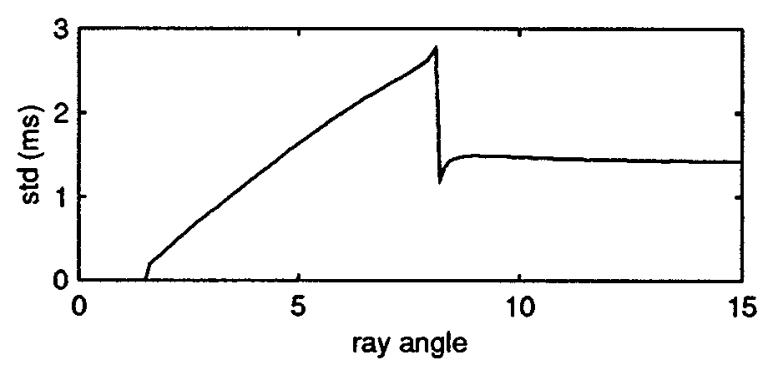

FIG. 5. Standard deviation of ray travel time perturbations from Eq. (8). where $c$ is the index of the center mode of the sum and $2 l+1$ is the number of modes included in the sum. The center mode is chosen as the mode with the maximum travel-time variability. This is not necessarily the mode with the angle closest to the angle associated with the ray that has maximum variability as shown earlier. The number of modes is chosen as a trade-off between an angular window that is too large if $l$ is large, or has poor constructive interference if $l$ is small. Although approximate analytical methods for choosing $l$ are given in Tindle and Guthrie's paper based on phase difference limit of $\pi / 2$ between modes for constructive interference, this method of choosing $l$ is awkward except when applied to very simple sound-speed profiles.

An alternate method of choosing the best number of modes to include in the sum is to examine the interference pattern for a range of $l$ 's and decide which gives the "narrowest" ray. This procedure is quantified using a concept similar to that of a directivity index in beamforming. The normalized inference pattern is integrated over a given area and the value of $l$ that results in the minimum integrated response is considered to give the best representation of the ray:

$$
l_{\text {optimum }}=\min _{l}\left(\iint \frac{|p(r, z)|^{2}}{\left|p_{\max }\right|^{2}} d r d z\right) .
$$

In Fig. 6(a) the modal interference pattern for the $300-\mathrm{Hz}$ case is displayed. Here $l_{\text {optimum }}=1$ so three modes are used in the sum. The ray with maximum variability has an angle of $8.1^{\circ}$ and the center mode has an angle of $9.4^{\circ}$. The modal interference pattern is also calculated for a frequency of $2500 \mathrm{~Hz}$ [Fig. 6(b)]. For this case $l_{\text {optimum }}=11$ so 23 modes are used, and the center mode has an angle of $8.19^{\circ}$.

\section{TRAVEL-TIME VARIANCE OF THE TRUNCATED MODAL SUM}

From Fig. 6, it is evident that the truncated modal sum does represent a ray. In order to find the travel-time variance of the truncated modal sum $\left\langle\delta t_{\Sigma}^{2}\right\rangle$, a definition for the arrival time perturbation $\left(\delta t_{\Sigma}\right)$ due to a sum of modes is required. The travel-time perturbation for a single mode is well defined by the stationary point of the phase integral. The definition used here for the travel-time perturbation due to a sum of modes is a weighted average of the individual modal travel-time perturbations:

$$
\delta t_{\Sigma w}=\sum_{m=c-l}^{c+l} W_{m} \delta t_{m}
$$

The subscript $\Sigma w$ in the notation $\delta t_{\Sigma w}$ indicates a weighted average sum.

The weighted average function $W$ is normalized by

$$
W_{m}=w_{m}\left(\sum_{m=c-l}^{c+l} w_{m}\right)^{-1} \text {. }
$$

The weighting function $w$ is a Hamming window centered at the index $l$ of the center mode. The choice of a Hamming window is arbitrary; other windowing functions will give similar behavior. The motivation for using a weighted sum 
instead of a straight average is that the contributions of the outlying modes to the modal representation of a ray are less significant. A straight average would also display abrupt behavior as the modal travel-time variance resonant peak is included in the sum while the weighted sum has smooth behavior. A potential problem with this definition of the travel-time perturbation due to a finite sum of modes is that it sums the individual modal travel-time perturbations in an incoherent manner. It does not account for the exact phase interferences that may occur in such a sum. The variance of the weighted average sum $\left\langle\delta t_{\Sigma w}^{2}\right\rangle$ is compared to the variance of a coherent sum of modal travel-time perturbations $\left\langle\delta t_{\Sigma c}^{2}\right\rangle$ in Appendix A. It is found that the variances are similar using both methods.

Using the weighted sum definition of the travel-time perturbation the modal function $G_{m}(z)$ in Eq. (6) is replaced by a weighted average of $G(z)$ over the $2 l+1$ modes. The expression relating the travel-time variance due to a trun-

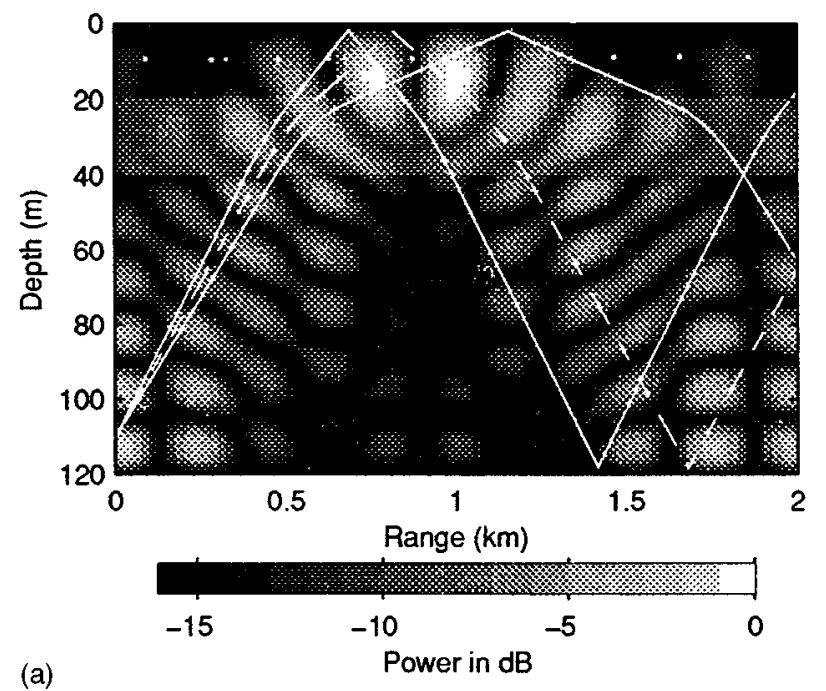

(a)

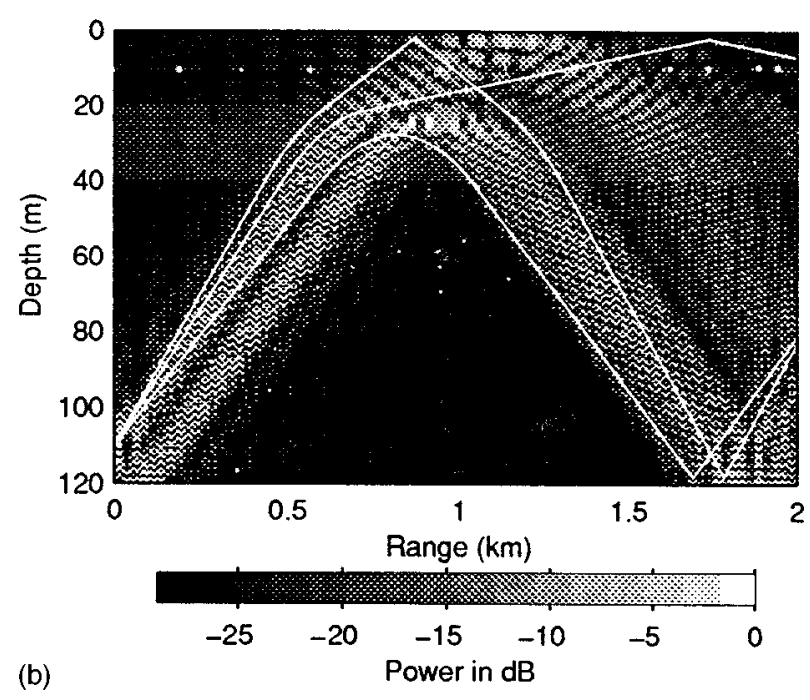

FIG. 6. Modal interference patterns for the truncated sum of modes with superimposed rays. The three rays that are plotted are the rays with the same grazing angle as the lowest mode, the center mode, and the highest mode in the sum. In both (a) and (b) power is normalized so the maximum is $0 \mathrm{~dB}$, and power is scaled by the range so cylindrical spreading is not visible. The source is placed at a depth of $110 \mathrm{~m}$ in both cases. (a) $f=300 \mathrm{~Hz}$, (b) $f=2500 \mathrm{~Hz}$.

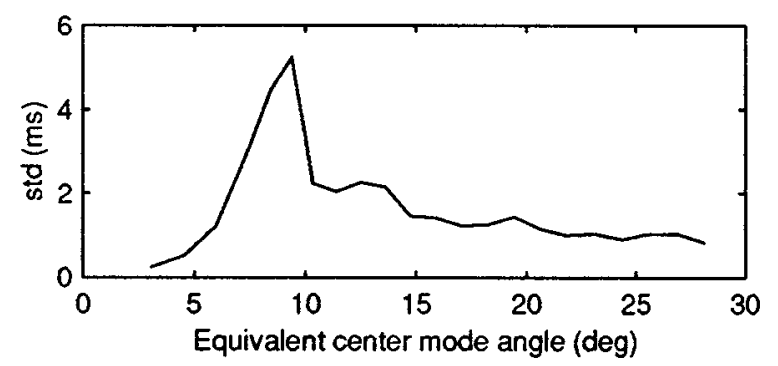

FIG. 7. Standard deviation of truncated modal sum travel-time perturbations $f=300 \mathrm{~Hz}$

cated sum of modes to the variance of the internal wave amplitudes is

$$
\left\langle\delta t_{\Sigma w}^{2}\right\rangle=\sum_{n}\left\langle a_{n}^{2}\right\rangle\left(R \sum_{m=c-l}^{c+l} W_{m} \int_{0}^{D} \psi_{n}(z) S_{z} G_{m} d z\right)^{2} .
$$

This expression is evaluated for the $300-\mathrm{Hz}$ case and the results are displayed in Fig. 7. While the maximum traveltime standard deviation due to the individual modes is 9.0 $\mathrm{ms}$, the maximum standard deviation due to the modal representation of a ray at $300 \mathrm{~Hz}$ is $5.23 \mathrm{~ms}$. This is closer to the ray maximum standard deviation of $2.8 \mathrm{~ms}$.

\section{CONVERGENCE AT HIGHER FREQUENCIES}

At higher frequencies the modal sum can more accurately represent a ray since the number of modes in a given angular window increases. As the mode shape oscillates at higher frequencies it is better able to fit half a vertical oscillation into the layer of nonzero sound-speed gradient. While the ray maximum variance had a frequency-independent geometric limit, the maximum "resonant" individual modal travel-time variance continues to increase with increasing frequency.

The travel-time standard deviations of the individual modes are compared to the travel-time standard deviations of the truncated sum for a frequency of $2500 \mathrm{~Hz}$ in Figs. 8 and 9. At this frequency the maximum travel-time standard deviation of the 47 th mode with a grazing angle of $8.19^{\circ}$ is 195 $\mathrm{ms}$. This is two orders of magnitude greater than the ray maximum of $2.8 \mathrm{~ms}$ and an order of magnitude greater than the maximum modal standard deviation of $9.0 \mathrm{~ms}$ at $250 \mathrm{~Hz}$. The modal grazing angle is approaching that of the most perturbed ray $\left(8.1^{\circ}\right.$.) The maximum travel-time standard deviation due to the truncated sum modal representation of a

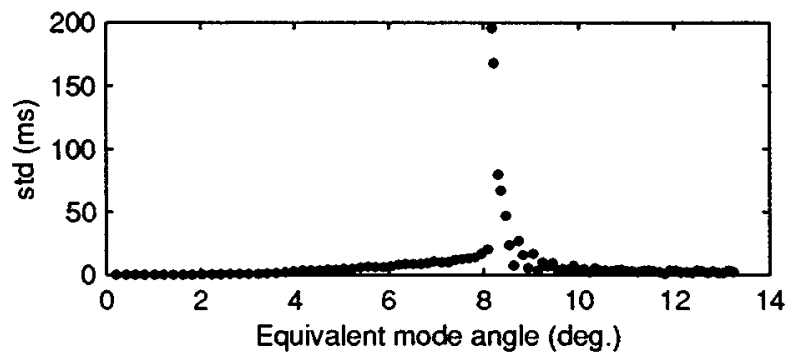

FIG. 8. Standard deviation of individual modal travel-time perturbations for $f=2500 \mathrm{~Hz}$. 


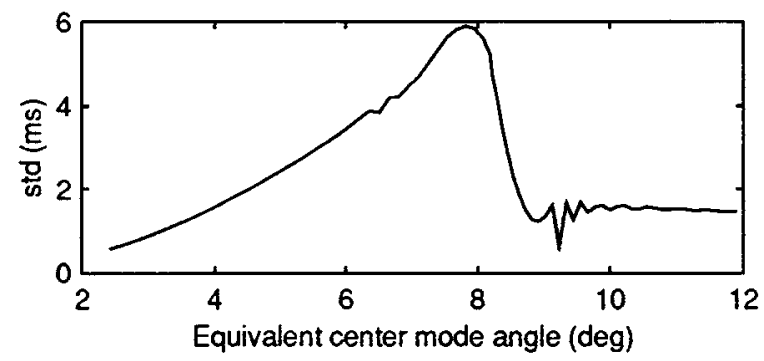

FIG. 9. Standard deviation of truncated modal sum travel-time perturbation for $f=2500 \mathrm{~Hz}$.

ray $(5.9 \mathrm{~ms})$ at $2500 \mathrm{~Hz}$ does not show a similar dramatic increase (Fig. 9). It is the same order of magnitude as the ray maximum.

The shape of the modal representation of a ray traveltime standard deviation versus angle curve (Fig. 9) is also similar to the ray travel-time standard deviation versus angle curve (Fig. 5). Both curves have a upward sloping region between $2^{\circ}$ and $8^{\circ}$ where the modal/ray energy is propagating partially through the gradient region. The maximum perturbations, which occur when the turning points are near the top of the gradient layer, are followed by regions of rapid decrease in both curves as the energy is able to propagate through the gradient layer. Once the energy has propagated through the gradient layer, both curves are relatively flat, with standard deviations near $1.5 \mathrm{~ms}$.

The maximum travel-time standard deviations for both the modal representation of a ray and the individual modes are calculated over the range of frequencies from 250 to $5000 \mathrm{~Hz}$ at intervals of $10 \mathrm{~Hz}$ (Fig. 10). While the modal resonant standard deviation continues to increase with higher frequencies, the standard deviations due to the modal representation of ray converge to a value of $5.9 \mathrm{~ms}$. Although this is slightly larger than the ray maximum of $2.9 \mathrm{~ms}$, it is of the same order of magnitude and, as shown in Appendix A, the definition used for the travel-time perturbation due to a sum
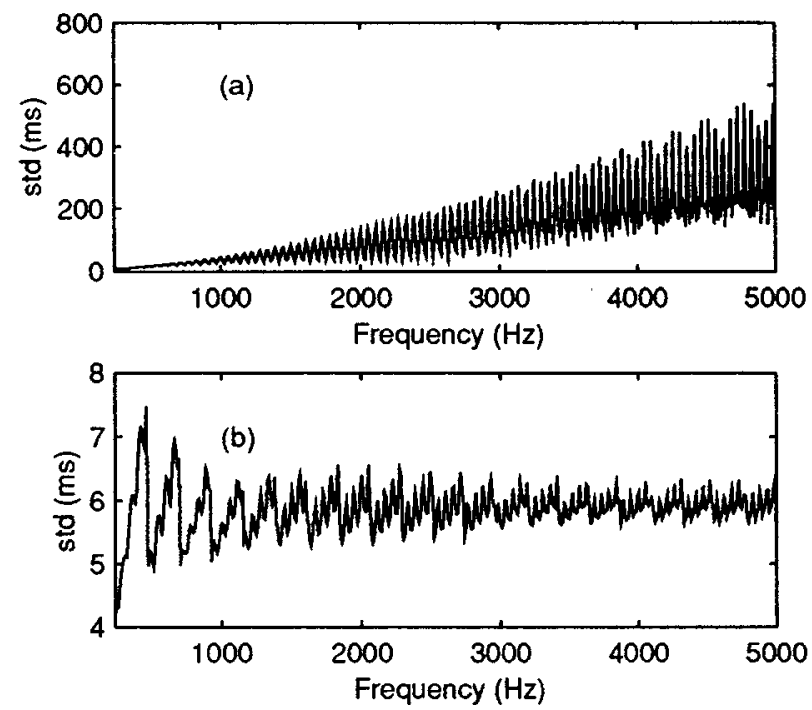

FIG. 10. (a) Maximum of the individual mode standard deviations versus frequency. (b) Maximum of the truncated sum standard deviation versus frequency. of modes is a conservative estimate in the lower frequency range. It will in fact overestimate the actual travel-time variance due to a sum of modes.

\section{CONCLUSIONS}

The central point of this paper is that the travel-time variance, due to internal waves, of a truncated sum of acoustic modes which sum to form a ray path is consistent with the travel-time variance of a geometric ray. The individual modal maximum travel-time variance continues to increase with increasing frequency as the geometry of the vertical oscillations matches the geometry of the internal wave layer. The maximum ray travel-time variance is limited by the curvature of the ray within the internal wave layer, independent of frequency.

\section{ACKNOWLEDGMENTS}

I would like to thank all the authors of the BSPF paper for the motivation for this work. Thanks especially go to Jim Lynch for the useful discussions. Financial support for the author was provided in part by the Office of Naval Research fellowship program and in part by ONR Grant No. N0001491-J-1246. This is WHOI contribution No. 8989.

\section{APPENDIX A}

The definition used in this paper for the travel-time perturbation due to a sum of modes does not account for the constructive or destructive interferences that may occur in the pressure signal of such a sum. An alternate definition of travel-time perturbations due to a sum of modes can be written by considering the energy centroid of a coherent sum of a group of modal pulses, each one delayed by the modal perturbation due to the internal waves. The modal pulse shape used in this comparison is a Gaussian shaped pulse of length $T_{l}$ :

$$
p(t)=\sum_{m} \exp \left[\frac{1}{2}\left(\frac{t-\delta t_{m}}{T_{l}}\right)^{2}\right] \exp \left(i \omega\left(t-\delta t_{m}\right)\right) .
$$

The arrival time perturbation is found from the energy centroid of Eq. (A1):

$$
\delta t_{\Sigma c}=\int_{-\infty}^{\infty} t\left|p^{\prime}(t)\right|^{2} d t
$$

The subscript $\Sigma c$ indicates a coherent sum as opposed to the weighted average incoherent sum $(\Sigma w)$ used in Sec. IV. $p^{\prime}(t)$ is $p(t)$ normalized so that

$$
\int_{-\infty}^{\infty}\left|p^{\prime}(t)\right|^{2} d t=1
$$

The modal delays ( $\left.\delta t_{m}\right)$ that are combined nonlinearly in Eq. (A1) to determine the coherent sum time perturbation $\left(\delta t_{\Sigma_{c}}\right)$ are functions of the random internal wave modal coefficients 


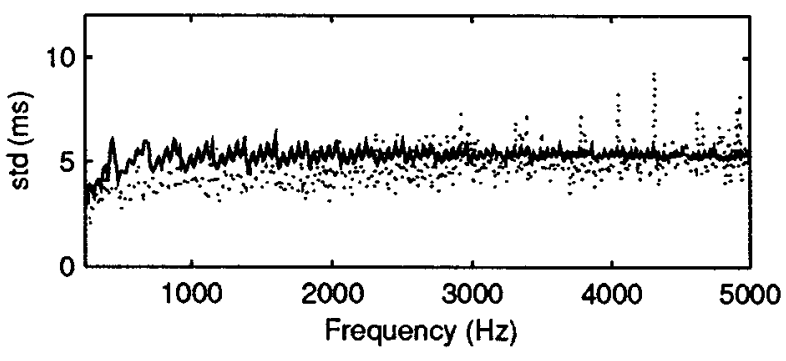

FIG. A1. Comparison of maximum travel-time standard deviation due to the incoherent sum (solid line) and coherent sum (dotted line).

$\left(a_{n}\right)$. Thus the variance of $\delta t_{\Sigma c}$ can be calculated by

$$
\begin{aligned}
\left\langle\delta t_{\Sigma c}^{2}\right\rangle= & \int_{-\infty}^{\infty} \cdots \int_{-\infty}^{\infty} \delta t_{\Sigma c}^{2}\left(a_{1}, \ldots, a_{n}\right) \\
& \times P\left(a_{1}, \ldots, a_{n}\right) d a_{1} \cdots d a_{n},
\end{aligned}
$$

where $P\left(a_{1}, \ldots, a_{n}\right)$ is the probability density function of the internal wave amplitudes. As stated in Sec. II A, statistics are available for only the mean and the variance of $P$. Thus $P$ is modeled as a Gaussian density with zero mean and variance equal to $\left|A_{n}(\omega)\right|^{2}$. It is very computationally intensive to calculate Eq. (A4) over the entire set of internal wave modes for a range of acoustic frequencies; thus only the first internal wave mode is used to compare the coherent sum variance to the weighted average sum variance [Eq. (14)]. This reduces Eq. (A4) to a one-dimensional integral. In Fig. A1 it can be seen that the variance of the coherent sum is consistently lower than the variance of the incoherent sum below 3000 $\mathrm{Hz}$ and of the same magnitude between 3000 and $5000 \mathrm{~Hz}$. The incoherent sum standard deviation with only one internal wave mode converges to a value of $5.4 \mathrm{~ms}$ at higher frequencies, while the mean of the coherent sum standard deviation is $4.2 \mathrm{~ms}$ in the region $500-2800 \mathrm{~Hz}$ and converges to $5.1 \mathrm{~ms}$ at high frequency.

\section{APPENDIX B}

\section{Approximations}

In this Appendix the validity of several of the approximations used in this paper and Lynch et al.'s BSPF paper to determine travel-time perturbations due to internal waves are examined. Some directions for improvement are also suggested.

\section{a. Fixed gradient layer}

The first approximation is in the method [Eq. (6): $\left.S_{z}=d c_{p} / d z\right]$ used to relate the perturbations in sound speed $\delta c$ to displacements $\xi$ from the internal waves:

$$
\delta c(z)=S_{z}(z) \xi(z) .
$$

In the sound-speed profile used in this paper this expression effectively fixes the gradient layer in place and only allows perturbations in sound speed within the stationary layer. This expression is exact for only a constant gradient sound-speed profile, but is also fairly accurate for small internal wave amplitudes with gradient layers thick compared to the depth of the waveguide. For thin gradient layers (the extreme case being a two-layer sound-speed profile with constant veloci- ties in each layer) and large internal wave amplitudes this approximation is not accurate. An exact method of relating sound-speed changes to displacements from the internal waves is

$$
c_{1}(z)=c_{p}(z-\xi(z))
$$

where $c_{1}(z)$ is the perturbed sound-speed profile. The internal wave amplitudes and mode shapes in the body of the paper cause displacements of up to $20 \mathrm{~m}$; thus the fixed layer approximation may cause errors. These errors are discussed in Sec. 2 of this Appendix. For large internal wave displacements the assumptions leading to a modal solution for the internal wave displacements are not fulfilled.

\section{b. Linear perturbation acoustics}

The second approximation in both papers is the linear first-order perturbation approach used to relate perturbations in the sound speed to travel-time perturbations. In the modal case this involves determining the travel-time perturbation from the perturbation in horizontal wave number using the original mode shapes [Eqs. (6) and (7)]. The mode shapes of the perturbed sound-speed profile are not calculated. For the modal case an exact expression for travel-time perturbations of mode $m$ is

$$
\begin{aligned}
\delta t_{m}= & R\left(\frac{\omega}{k_{r m}} \int_{0}^{D} \frac{\phi_{m}^{2}(z)}{\rho(z) c_{0}(z)} d z\right. \\
& \left.-\frac{\omega}{k_{r m}^{\prime}} \int_{0}^{D} \frac{\phi_{m}^{\prime 2}(z)}{\rho(z) c_{1}(z)} d z\right),
\end{aligned}
$$

where $\phi^{\prime}$ and $k_{r m}^{\prime}$ correspond to the perturbed sound-speed profile $c_{1}(z)$. This expression involves calculating two sets of mode shapes and horizontal wave numbers.

In ray theory the linear first-order perturbation approach assumes that ray paths remain stationary and higher-order terms in the perturbation expansion can be ignored. Exact travel-time perturbations for the ray case can be expressed as

$$
\delta t=\int_{\Gamma_{0}} \frac{1}{c_{0}} d s-\int_{\Gamma_{1}} \frac{1}{c_{1}} d s
$$

where the second integral is evaluated along the new ray path determined by the perturbed sound-speed profile. This exact expression requires twice the computation of the linear expression, and does not define a linear relation between sound-speed perturbations and travel time.

\section{Evaluation of approximations}

To investigate the difference between the exact solutions and the linear approximations, two example simulations were performed.

\section{a. Three-layer ocean model}

In the first simulation, the three-layer sound-speed and buoyancy frequency profile used in the body of this paper is used with both the approximate and exact expressions. Only one internal wave mode is used as in Appendix A to simplify the calculation of the travel-time variance. The amplitudes of 
the internal waves are the same as were used in the body of the paper for Fig. B1 and are reduced by a factor of 10 for Fig. B2.

Although the maximum ray travel-time standard deviations are similar for both the approximate and exact solutions with large internal wave amplitudes, the shape of the curves and values other than the maximum are quite different. The linear solution has a peak at an angle of $8^{\circ}$ as the turning point of the ray approaches the top of the layer. The exact solution is dominated by the rising and falling of the gradient layer (which is held fixed in the approximate solution.) Rays that do not penetrate through the gradient layer into the upper layer show little travel-time variance as they encounter the same sound speeds along their paths. Rays steeper than $8^{\circ}$ that do penetrate into the upper layer show a greater variability due to the changing thickness of the faster soundspeed upper layer. This difference in physics for the exact solution and the approximate solution indicates that for thin gradient layers relative to the waveguide thickness, acoustic modeling based on a moving gradient layer may be more appropriate. A simple two-layer model may capture most of the physics for the thin gradient layer with large internal wave amplitudes.

Comparing the ray solution for high amplitude internal waves (Fig. B1 top) to the ray solution for low amplitude internal waves (Fig. B2 top) shows little change in shape of the curve as the internal wave amplitude changes. While not deceasing by an exact factor of 10 as the linear solution does, the exact solution does display a nearly linear behavior in response to changes in internal wave amplitude.

The modal approximate and exact solutions display substantial differences for large internal wave amplitudes (Fig. B1). The linear solution shows sharp resonances and nulls as the $G$ function [Eq. (7)] oscillations match or mismatch the
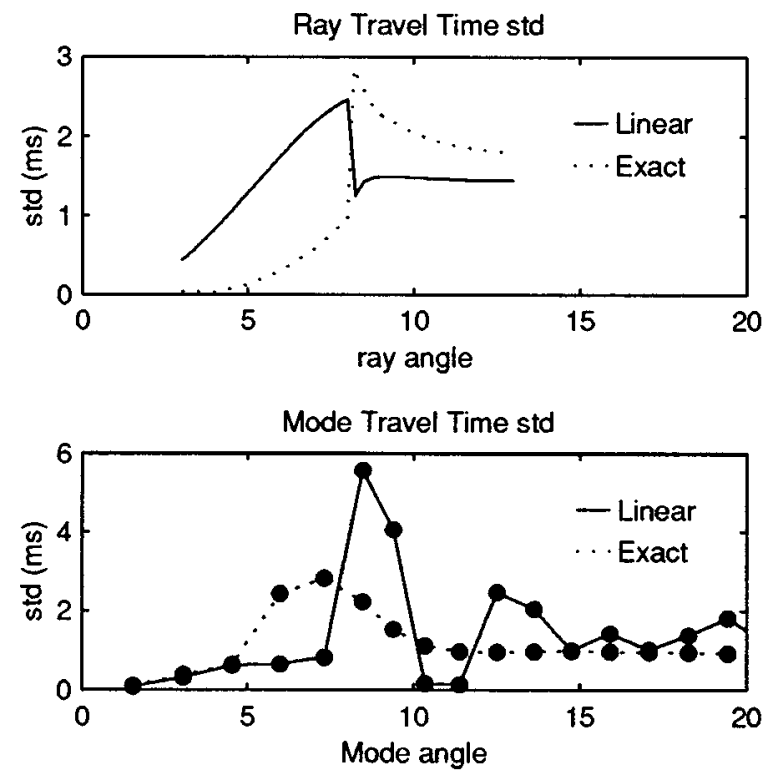

FIG. B1. Comparison of exact and linear theory for travel-time standard deviations using the three-layer waveguide considered in the body of the paper with one internal wave mode. Maximum vertical displacements of 20 $\mathrm{m}$. The thick dots represent the individual acoustic modes. Acoustic frequency is $300 \mathrm{~Hz}$.
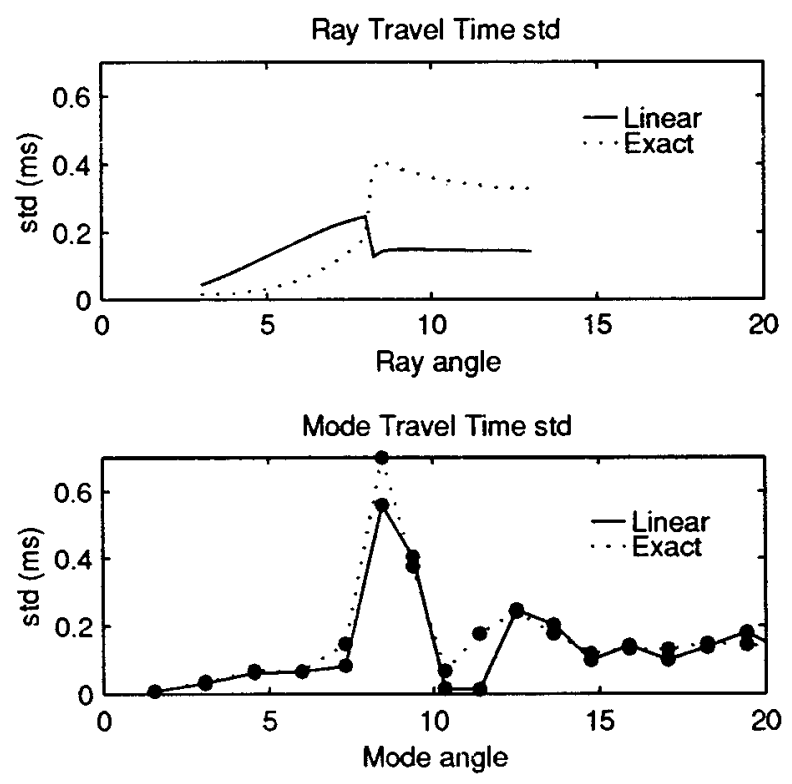

FIG. B2. Comparison of exact and linear theory for travel-time standard deviations using the three-layer waveguide considered in the body of the paper with one internal wave mode. Maximum displacement reduced to $2 \mathrm{~m}$. Acoustic frequency is $300 \mathrm{~Hz}$.

scale of the gradient layer. The exact solution displays a smoother behavior, and also shows a smaller peak as the turning point of the mode interacts with the gradient layer. This smoother behavior is due to the changing thickness of the gradient layer with large internal wave amplitudes. With small internal wave amplitudes (Fig. B2 bottom) the modal exact and linear solutions are similar (Fig. B2), because the fixed layer approximation is not grossly violated.

Figure B3 examines the frequency dependence of the exact modal solution for both large and small internal waves. With large amplitudes the travel-time standard deviation is not frequency dependent because of the changing thickness
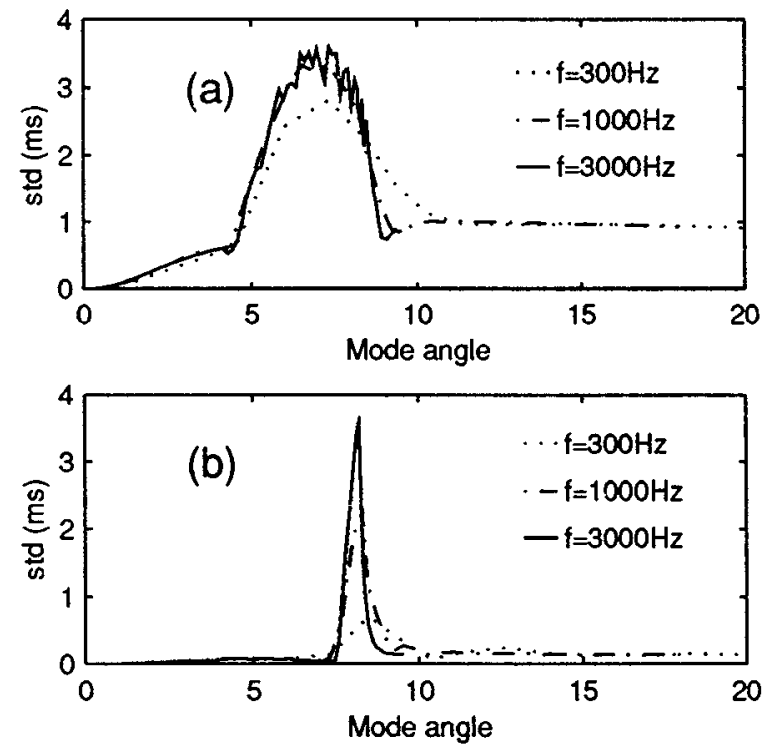

FIG. B3. Exact theory for travel-time standard deviations for three different acoustic frequencies using the three-layer ocean model and one internal wave mode. (a) Maximum internal wave displacements of $20 \mathrm{~m}$. (b) Maximum displacements of $2 \mathrm{~m}$. 
of the gradient layer [Fig. B3(a)]. For small amplitudes the layer does not change thickness and the resonant response seen in the linear solution is also present in the exact solution [Fig. B3(b)]. The large amplitude solution also displays a peak spread over a larger mode angle due to the changes in horizontal wave number as the lower constant sound-speed layer changes thickness.

\section{b. Constant linear gradient ocean model}

Simulations using the three-layer ocean model are sensitive to errors due to both the fixed layer approximation and the nonlinearity of the acoustic travel-time variations. To examine only the effects of the acoustic linear perturbation methods an ocean model with a constant linear gradient sound-speed profile is used. For this sound-speed profile the fixed layer approximation [Eq. (B1)] is an exact method of representing sound-speed changes due to vertical displacements. Thus any difference between the exact solution and the linear solution will be due to the linearization of the travel-time perturbations. Figure B4 displays the travel-time perturbation due to a water column vertical displacement equal to the rms value of the first internal wave mode. This figure displays only the travel-time perturbation and not the standard deviation of this quantity. Thus this tests the evaluation of travel-time perturbations only and not the moment generating process of these perturbations.

Figure B4 shows that the exact modal solution agrees well with the linearized form. This is encouraging since it
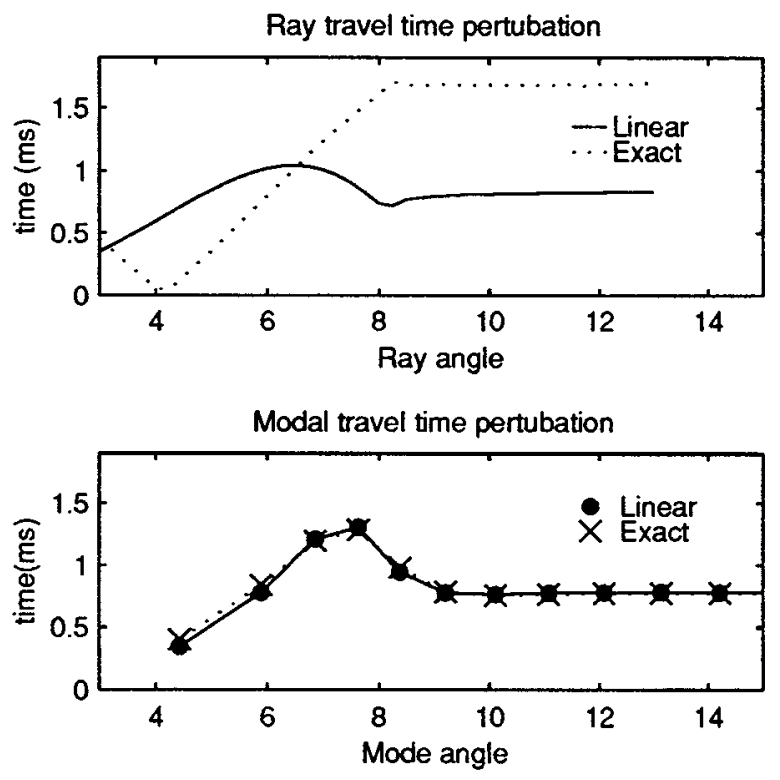

FIG. B4. Comparison of exact and linear theory for travel-time perturbations using a constant linear gradient sound-speed profile and one internal wave mode. The thick dots and $\times$ 's represent the individual acoustic modes. Acoustic frequency is $300 \mathrm{~Hz}$. implies that to improve our estimates of modal travel-time variations only the fixed layer approximation must be improved, and the linear acoustics approximation may be retained. The exact and linearized ray solutions do not agree as well. This is largely due the fact that there is substantial displacement of the ray paths due to the sound-speed profile variations. Thus to more accurately model the ray travel-time variation, the displacements of ray paths will need to be considered.

\section{Implications for equivalence of rays and modes}

The implications of the results in this Appendix on the equivalence of modal and ray travel times demonstrated in the body of the paper can be seen from carefully examining Figs. B1 and B3. The purpose of the paper is to show that the strong resonant peak in the modal travel-time variance can be shown to be equivalent to the maximum ray travel-time variance by considering a truncated sum of modes. As seen in Fig. B1 the maximum ray travel-time standard deviation is similar for both linear and exact theory, although the shape of the curve varies somewhat. While the exact modal theory does not show the frequency-dependent resonant response for large internal wave amplitudes, for small amplitudes it is clearly visible (Fig. B3). Thus the reduction in travel-time variance, achieved by considering the truncated sum of modes, is necessary to show equivalence with the ray solution when the internal wave amplitudes are small. When the internal wave amplitudes are large the travel-time standard deviations of the exact ray and modal solution are roughly similar. Because the resonances do not appear with large internal wave amplitudes, the truncated sum is not required to show equivalence. Thus performing the truncated sum on the exact solution will give a similar result to the exact solution itself since there are no sharp resonances.

${ }^{1}$ C. Garrett and W. Munk, "Internal waves in the ocean," Ann. Rev. Fluid Mech. 11, 339-69 (1979).

${ }^{2}$ S. Flatté, R. Dashen, W. Munk, K. Watson, and F. Zachariasen, Sound Transmission through a Fluctuating Ocean (Cambridge U.P., Cambridge, 1979).

${ }^{3}$ H. H. Essen, F. Schirmer, and S. Sirkes, "Acoustic remote sensing of internal waves in shallow water," Int. J. Remote Sens. 4, 33-47 (1983).

${ }^{4}$ J. F. Lynch, J. Guoliang, R. Pawlowicz, D. Ray, C. S. Chiu, J. Miller, R. H. Bourke, R. Parson, A. Pluedemann, and R. Muench, "Acoustic travel time perturbations due to the shallow water internal waves and internal tides in the Barents sea polar front: Theory and experiment," J. Acoust. Soc. Am. 99, 803-821 (1996).

${ }^{5} \mathrm{C}$. T. Tindle and K. M. Guthrie, "Ray effects in the normal mode approach to underwater acoustics," J. Sound Vib. 47(3), 403-413 (1976).

${ }^{6}$ A. E. Gill, Atmosphere-Ocean Dynamics (Academic, San Diego, 1982), pp. 154-168 and pp. 256-260.

${ }^{7}$ F. B. Jensen, W. A. Kuperman, M. B. Porter, and H. Schmidt, Computational Ocean Acoustics (American Institute of Physics, Woodbury, NY, 1994), pp. 314-315. 